Z Epileptol 2013 · 26:5-5

DOI 10.1007/s10309-012-0297-8

Online publiziert: 16. Januar 2013

(c) Springer-Verlag Berlin-Heidelberg 2013

\title{
U. Stephani
}

Klinik für Neuropädiatrie, Universitätsklinikum Schleswig Holstein Campus Kiel

\section{Beiträge des Königsteiner Arbeitskreises (für Epileptologie)}

Im Februar 2012 hat der Königsteiner Arbeitskreis (für Epileptologie) zum 37. Mal getagt. Er wurde 1974 als deutschsprachiger Arbeitskreis von epileptologisch orientierten Neuropädiatern gegründet. Tagungsort ist jahrzehntelang ein Hotel direkt in Königstein gewesen, jetzt ganz in der Nähe im Taunus, Tagungszeit meist Ende Februar. Für ca. $1 \frac{1}{2}$ Tage findet sich der geschlossene Kreis der Kinder- und Erwachsenenepileptologen, Grundlagenwissenschaftler und Psychiater zusammen, um aktuelle Themen der Epileptologie zu besprechen und sich gegenseitig interessante Kasuistiken zu präsentieren. Die Tagung wird von Anbeginn an durch die Firma Desitin unterstützt.

Seit seinem Bestehen sind zahlreiche Berichte, Empfehlungen und Einschätzungen von diesem Kreis publiziert worden, so auch in letzter Zeit Publikationen zu neuen Antiepileptika, zum Absetzen von Antiepileptika, zum plötzlichen unerwarteten Tod bei Epilepsie („sudden unexpected death in epilepsy“, SUDEP), zum therapierefraktären Status epilepticus sowie zur neuen Klassifikation epileptischer Syndrome und Anfälle. Diese Tradition wird mit dem vorliegenden Heft der Zeitschrift für Epileptologie fortgesetzt, in dem interessante Kasuistiken und Beiträge von der Tagung 2012 wiedergegeben sind.

Die Autoren nahmen die Einladung von Herrn Prof. Steinhoff gern auf, ihre Beiträge (mit den jeweils beteiligten Mitarbeitern) in diesem Heft zu publizieren. Neben einzelnen Kasuistiken sind es
Übersichtsarbeiten zur pharmakologischen Therapie, zu neuen neurophysiologischen Methoden und zu Klassifikationsfragen, auf die in diesem Heft eingegangen wird.

Ich danke allen Autoren dieses Schwerpunkthefts für das Zusenden der Manuskripte.

\section{Ulrich Stephani}

\section{Korrespondenzadresse}

Prof. Dr. U. Stephani

Klinik für Neuropädiatrie, Universitätsklinikum

Schleswig Holstein Campus Kiel

Schwanenweg 20, 24105 Kiel

stephani@pedneuro.uni-kiel.de

Interessenkonflikt. Der korrespondierende Autor weist auf folgende Beziehung hin: Unterstützung durch die Fa. Desitin. 